\section{ÜBRIGENS}

... ist bei Diabetesausbruch in der Adoleszenz die Gefahr für Risikoverhalten und frühen Tod stark erhöht. Von 719 Typ-1-Diabetikern in Norwegen, die als Jugendliche oder junge Erwachsene erkrankt waren, starb jeder Fünfte binnen 36 Jahren. Außer akuten und chronischen Diabeteskomplikationen waren besonders Alkoholmissbrauch, Unfälle und Suizide die Ursachen.

Diab Care 2017;40:38.

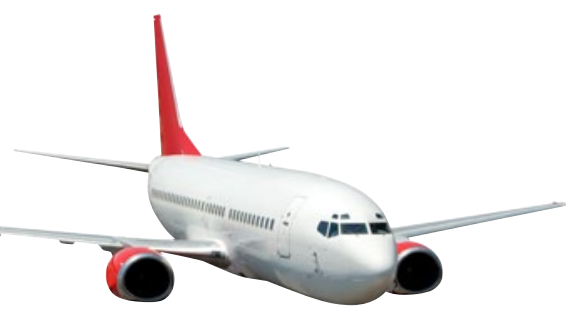

.... können insulinpflichtige Berufspiloten in Großbritannien eine Fluglizenz erwerben. Das Risiko ist offenbar gering, wurde beim 52. Kongress der European Association for the Study of Diabetes (EASD) berichtet. So gab es bei 26 Piloten in 18 Monaten mit 4900 Flugstunden keine Zwischenfälle. Von 8897 Zuckermesswerten lagen $96 \%$ im grünen $(90-270 \mathrm{mg} / \mathrm{dl})$ und nur $0,2 \%$ im roten Bereich ( $<72 \mathrm{mg} / \mathrm{dl})$.

Punkte sammeln in der Sommerakademie mit e.Med

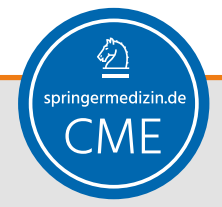

Auch 2017 haben wir in der Sommerakademie ausgewählte CME-Kurse für Ihre hausärztliche Fortbildung zusammengestellt.

Zum Thema "Diabetes" finden Sie unter http://bit.ly/2hFwgdK

aktuell den Kurs

\section{"Strategien zur Gewichtsreduktion} bei Typ-2-Diabetes"

Zur Teilnahme benötigen sie ein e.Med-Abo, mit dem sie Zugang zu allen Inhalten auf SpringerMedizin.de erhalten.

Nutzen Sie die Sommerzeit um CME-Punkte zu sammeln und um sich schnell und effizient fortzubilden. Testen Sie SpringerMedizin.de 30 Tage lang kostenlos und unverbindlich mit dem e.Med-Abo: http://bit.ly/2vhm8el

Kardiovaskuläre Mortalität

\title{
Antidiabetika: Nun ein Viertes mit Herzschutz
}

Nach Empagliflozin, Liraglutid und Semaglutid ist nun auch für das Antidiabetikum Canagliflozin bewiesen, dass sich damit die Inzidenz kardiovaskulärer Ereignisse reduzieren lässt.

Dass es gelingen könnte, durch eine Therapie mit blutzuckersenkenden Antidiabetika das Risiko für kardiovaskuläre Erkrankungen bei Patienten mit Typ-2-Diabetes (T2D) substanziell zu reduzieren, galt lange Zeit als fraglich. Das änderte sich mit der EMPA-REG OUTCOMEStudie, in der der SGLT-2-Hemmer Empagliflozin bei Patienten mit T2D und erhöhtem kardiovaskulärem Risiko die kardiovaskuläre Mortalität signifikant verringerte. In der LEADER- und SUSTAIN-6-Studie waren in der Folge auch die GLP-1-Agonisten Liraglutid und Semaglutid in Sachen kardiovaskuläre $\mathrm{Ri}$ sikoreduktion erfolgreich.

Belege für eine kardioprotektive Wirkung gibt es jetzt auch für den SGLT2-Hemmer Canagliflozin aus der Cana- gliflozin Cardiovascular Assessment Study (CANVAS) [1]. Der primäre Studienendpunkt in CANVAS ist die Kombination der Ereignisse kardiovaskulärer Tod, Herzinfarkt und Schlaganfall. Ebenso wie durch Empagliflozin in EMPA-REG-OUTCOME wurde auch in CANVAS das relative Risiko für diesen kombinierten Endpunkt durch Canagliflozin signifikant um 14\% im Vergleich zu Placebo reduziert. In Deutschland wurde Canagliflozin allerdings wegen der negativen Nutzenbewertung durch den GBA (kein nachgewiesener Zusatznutzen) vom Markt genommen.

\section{Herzwirksame Arzneien}

Insgesamt scheint die blutzuckersenkende Wirkung dieser neueren Antidiabetika für den gezeigten prognostischen Nutzen ohne Bedeutung zu sein. Sie präsentieren sich damit als herzwirksame Arzneien ähnlich den Statinen, ACE-Hemmern oder AT1-Rezeptorblockern.

ob/eis

\footnotetext{
1. NEJM 2017; online 12. Juni 2017,
}

DOI: 10.1056/NEJMoa1611925

\section{Adipositas}

\section{Remission nach bariatrischer Chirurgie}

Bei Adipositas-Patienten mit Diabetes lässt sich durch starke Gewichtsreduktion eine Remission der Zuckerkrankheit erreichen. Das Potenzial chirurgischer Eingriffe zeigt eine Analyse von Daten der SOS-Studie in Schweden: Von den 415 Patienten mit bariatrischer Chirurgie hatten noch $63 \%$ zwei Jahre nach Op eine Remission; das heißt, der Nüchternblutzucker lag ohne Antidiabetika unter $101 \mathrm{mg} / \mathrm{dl}$ [1]. Bei den 312 Patienten ohne Chirurgie hatten $14 \%$ eine Remission.

Über 90\% der Patienten mit Chirurgie, aber weniger als $40 \%$ der Kontrollpatienten, hatten nach zwei Jahren eine gute Blutzuckerkontrolle.

Fazit: Mit bariatrischer Chirurgie lässt sich Diabetes nicht unbedingt heilen, aber der Therapiebedarf wird stark reduziert. 